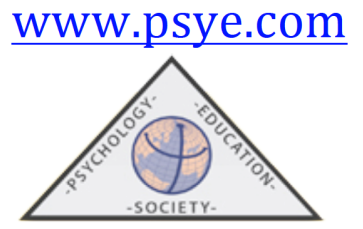

\title{
Mindfulness e investigación psicológica positivista: críticas y alternativas
}

\author{
Cornelio ÁGUILA
}

Universidad de Almería, España

(Recibido el 10 de Octubre de 2018, Aceptado el 25 de Noviembre de 2019)

\begin{abstract}
RESUMEN: En los últimos años, el fenómeno mindfulness está recibiendo una gran atención en el ámbito científico-académico, y su práctica es aceptada como una eficiente técnica para la mejora de diferentes trastornos psicológicos, habiendo sido integrada en las terapias de tercera generación. En su desarrollo científico, existe un claro predominio de las investigaciones de carácter positivista, que utilizan métodos racionalistas y técnicas de medición estandarizada. Dada la posición ontológica dual materialista del paradigma positivista, estas investigaciones presentan una visión reduccionista y limitada de mindfulness, al distanciarse de su naturaleza esencial no dualista, así como de sus fundamentos morales e implicaciones políticas. En este artículo, se señalan algunas de las cuestiones críticas de la investigación positivista sobre mindfulness en el campo de la psicología, y se muestran alternativas ontológicas y metodológicas más cercanas a su naturaleza original, defendiendo, para ello, el paradigma naturalista y los métodos de investigación cualitativos.
\end{abstract}

Palabras clave: mindfulness, psicología, positivismo, ontología, naturalismo

\section{Mindfulness and positivist psychological research: criticism and alternatives}

\begin{abstract}
In recent years, the mindfulness phenomenon is receiving great attention in the scientific-academic field, and its practice is accepted as an efficient technique for the improvement of different psychological disorders, having been integrated into third-generation therapies. In its scientific development, there is a clear predominance of positivist research, which uses rationalist methods and standardized measurement techniques. Given the dual materialistic ontological position of positivist paradigm, these investigations present a reductionist and limited view of mindfulness, distancing themselves from their essential non-dualistic nature, as well as their moral foundations and policy implications. In this paper, some of the critical questions of positivist research on mindfulness in the field of psychology are pointed out, and ontological and methodological alternatives closer to their original nature are shown, defending, for this, the naturalistic paradigm and the methods of qualitative research.
\end{abstract}

Keywords: mindfulness, psychology, positivism, ontology, naturalism

Correspondencia: Cornelio Águila Soto. Universidad de Almería, Ctra de Sacramento, s/n, 04120. La Cañada de San Urbano (España). Email: cornelio@ual.es 


\section{Introducción}

Desde finales del pasado siglo, en las sociedades occidentales se están introduciendo prácticas y técnicas de meditación orientales enfocadas a la mejora de la salud y la calidad de vida, en ámbitos como la medicina y la psicología clínica, la educación o la empresa. Particularmente, la psicología emplea el término mindfulness $(M F)$ para referirse a algunas de las prácticas de meditación budistas incorporadas a programas y a técnicas de diversa índole, dentro de las llamadas terapias de tercera generación (Ruiz y Callejón, 2014). La meditación y la práctica de la atención plena son comunes en vías espirituales orientales como el hinduismo, el tantra o el taoísmo, pero ha sido el budismo el que ha generado un método que ha estimulado el interés de los científicos occidentales (Cebolla y Campos, 2016). Mindfulness, término en inglés para la palabra Sati de la extinguida lengua pali, remite particularmente a la meditación vipassana del budismo (meditación en la atención plena) tal y como está siendo utilizado en el campo de la psicología (Miró, 2006), y suele traducirse al español como atención o consciencia plena. Sati refiere a una actividad no conceptual y no verbal basada en la atención plena estabilizada, lo cual permite que emerja un estado de consciencia no condicionada por las memorias autobiográficas, que proviene de nuestro sentir puro en la experiencia directa (Miró, 2006). En este sentido, $M F$ es un estado de consciencia pura, no conceptual y no verbal, libre de los filtros y del análisis del pensamiento, conectado a la contemplación y a la exploración de la experiencia directa en el presente (Kabat-Zinn, 2009; Siegel, 2010).

No obstante, ni el término inglés mindfulness ni los españoles atención o consciencia plena, recogen la profundidad del significado original, por cuanto no existe un término equivalente en la cultura occidental. Con ello, $M F$ hace referencia a vivir con plenitud el momento presente, mantener la atención plena en aquello que estamos experimentando sin atender al flujo de pensamiento ordinario que constantemente surge en nuestra mente. Vivir con atención plena implica estar en el presente con toda nuestra consciencia, con todos nuestros sentidos, con presencia (Kabat-Zinn, 2009).

Mindfulness ha sido incorporado a la psicología contemporánea como una técnica que permite gestionar más eficientemente ciertos procesos mentales relacionados con diferentes trastornos psicológicos (Bishop et al., 2004; Hervás, Cebolla y Soler, 2016), lo cual ha generado una enorme cantidad de investigaciones que señalan su eficacia en problemas como la ansiedad, el déficit de atención, la depresión, el estrés o el dolor crónico (v. Chiesa, Calati y Serretti, 2011; Goleman y Davidson, 2017; Keng, Smoski y Robins, 2011; Linares, Estévez, Soler y Cebolla, 2016; Ruiz y Callejón, 2014; Sansó et al., 2018; Spijkerman, Pots y Bohlmeijer, 2016; Strauss, Cavanagh, Oliver y Pettman, 2014; Yagüe, Sánchez-Rodríguez, Mañas, Gómez-Becerra y Franco, 2016). Asimismo, la práctica de la atención plena combinada con el fomento de la amabilidad (Cebolla, Enrique, Alvear, Soler y García-Campayo, 2017), la compasión (Campos et al., 2016; Cebolla et al., 2017; Simón, 2014) y la gratitud (Cebolla et al., 2017) se refuerzan mutuamente y conducen hacia resultados positivos sobre el bienestar y la felicidad, entendidos como un estado profundo de serenidad y plenitud mantenido en el tiempo, que nos permite gestionar mejor los distintos estados emocionales.

Sin embargo, la mayor parte de estas investigaciones son de carácter positivista (véase, p.e. Sansó et al., 2018; Spijkerman, Pots y Bohlmeijer, 2016; Strauss, Cavanagh, Oliver y (c) Psy, Soc, \& Educ, 2020, Vol. 12(1) 
Pettman, 2014; Yagüe et al., 2016), y, por tanto, se enfrentan a la paradoja de estudiar un fenómeno ontológicamente no conceptual y no dualista, como es $M F$, utilizando métodos basados en una visión dualista del mundo y a través de técnicas de medición estandarizada. Así pues, es posible que esta práctica milenaria se esté convirtiendo cada vez más en un mero ejercicio situado en una intervención psicológica (Mañas, 2009), en un filón para la investigación positivista o en una práctica de moda que desatiende sus fundamentos morales originales (Hyland, 2017). Este enfoque podría entrar en fuerte contradicción con su naturaleza original, pues la comprensión de $M F$ no es posible mediante técnicas y métodos exclusivamente racionalistas (Singh Sikh y Spence, 2016), por lo que podría estar perdiendo su significado profundo, al verse explotado, distorsionado o extraído de su nicho ecológico esencial (Williams y Kabat-Zinn, 2013).

El objetivo de este artículo es señalar algunas cuestiones críticas de la investigación positivista sobre $M F$ en el campo de la psicología, así como mostrar posibles alternativas más cercanas a su naturaleza original. Para ello, partiremos del análisis del constructo mindfulness desde la base de la psicología budista para, a continuación, centrarnos en algunos aspectos problemáticos y contradictorios de la investigación de carácter positivista. Finalmente, propondré alternativas científicas que nos permitan el estudio de la atención plena de una manera más profunda y más fiel a su esencia.

\section{¿QUÉ ES MINDFULNESS? EXAMINANDO EL CONSTRUCTO PSICOLÓGICO DESDE SU NATURALEZA ORIGINAL}

Comprender mindfulness en su profundidad, implica detenernos, necesariamente, en su naturaleza ontológica. Las principales tradiciones espirituales orientales no conciben el mundo y la vida desde la perspectiva materialista hegemónica de occidente, sino que, para ellos, la esencia de la realidad es una cualidad inmaterial, espiritual, que es irreductible y que impregna todos los seres, animados o no (Taylor, 2018). Aunque existen diversos términos para nombrarla según la tradición (p.e. brahmán en el hinduismo o tao en el taoísmo), todas ellas comparten la asunción de una realidad ontológica pura no dual, que podemos llamar consciencia universal, una fuerza espiritual que se manifiesta como materia y como mente, y se canaliza a través de los seres y las cosas (Spira, 2019). En este sentido, para el budismo esta fuerza espiritual o consciencia es la mente, la base universal de la experiencia, creadora de felicidad o sufrimiento. Siguiendo a Sogyal-Rimpoché (2006), para el budismo tibetano, existen dos aspectos destacables de la mente: la mente ordinaria o sem, y la naturaleza misma de la mente o Rigpa.

La mente ordinaria es la mente cargada de un flujo constante de pensamientos, emociones y sensaciones físicas; es ese estado mental que tiene una consciencia discriminatoria, posee sentido de la dualidad, aferrándose o rechazando la experiencia. Es la mente "que piensa, trama, desea, manipula, que monta en cólera, que crea oleadas de emociones y pensamientos negativos por los que se deja llevar" (Sogyal-Rimpoché, 2006, p. 77). En este nivel de consciencia, creamos un sentido de la identidad construido sobre la base de lo que pensamos y sentimos, produciendo una imagen sobre nosotros mismos: el ego. Es importante señalar aquí que este concepto remite a la visión de las principales filosofías 
orientales y no al concepto psicológico occidental. La identificación con el ego crea un marco de referencia a partir del cual percibimos y entendemos la realidad; es decir, el ego funciona como un filtro de valoración e interpretación de las experiencias, de una manera automática puesto que es, en gran parte, inconsciente (Miró, 2006). Se trata, siguiendo a Taylor (2018), de una especie de estado de sueño que crea, por un lado, un fuerte sentido de la individualidad y de separación dualista del mundo, y, por otro, una percepción automática e insensibilizada del mundo fenomenológico, consecuencia de la falta de atención hacia el sentir y hacia todo lo que nos rodea. Así, "la mente ordinaria es la presa incesante de las influencias exteriores, de las tendencias habituales y el condicionamiento [...] su energía se consume en una proyección constante hacia afuera" (Sogyal-Rimpoché, 2006, p. 77).

Luego está la naturaleza misma de la mente, Rigpa, su esencia más profunda que es la consciencia universal pura que no se ve afectada ni por el cambio ni por la muerte, y, de acuerdo con Sogyal-Rimpoché (2006), permanece "oculta en el interior de nuestra propia mente, nuestra sem, envuelta y oscurecida por el rápido discurrir de nuestros pensamientos y emociones" (p. 78). La naturaleza de la mente no se limita a nuestra mente, sino que es la naturaleza de todo y una fuente de comprensión en sí misma: "la consciencia primordial, pura y prístina que es al mismo tiempo inteligente, cognoscitiva, radiante y siempre despierta. Se podría decir que es el conocimiento del propio conocimiento" (p. 78).

En ocasiones tenemos experiencias que nos acercan a la naturaleza de la mente, "quizás inspiradas por alguna composición musical que nos induce a la exaltación, por la serena felicidad que a veces experimentamos en la naturaleza o por la situación más ordinaria de la vida cotidiana" (Sogyal-Rimpoché, 1996, p. 83). Sin embargo, lo cierto es que rara vez accedemos a la naturaleza de la mente puesto que nuestra vida cotidiana se desarrolla en un continuo, acelerado e inconsciente proceder, en comportamientos automáticos dentro del constante juego mental entre el pasado y el futuro que nos impide el acceso al presente. En este sentido, la atención plena nos puede poner en contacto con la naturaleza de la mente desde la vivencia de los fenómenos de manera directa, sin análisis o interpretaciones. Por ello, mindfulness es la base fundamental de la psicología budista.

Desde esta diferenciación entre la mente y su naturaleza profunda, la práctica de la atención plena nos puede permitir liberarnos del sufrimiento causado por la interacción automática de pensamientos y emociones, para acceder a otros niveles de consciencia de la realidad y, de este modo, integrar la meditación en la acción, en la vida cotidiana (Kabat-Zinn, 2009). $Y$ es aquí donde la práctica de $M F$ va indisociablemente unida a sus fundamentos éticos y políticos. La base de una vida ética y moral consciente incluye la generosidad, la confiabilidad, la amabilidad, la empatía, la compasión, la gratitud, la inclusión, la ecuanimidad, todas ellas cualidades de la mente y del corazón que amplían las posibilidades de bienestar para uno mismo, y tienen efectos beneficiosos sobre el mundo (Alvear, 2015; Cebolla et al., 2017; Hyland, 2017). Así pues, la práctica de $M F$ nos puede ayudar a superar el estado de la mente caótico, confuso e indisciplinado y, así, aliviar el sufrimiento y mejorar la convivencia. La experiencia en atención plena nos permite conectar con una consciencia no dual, no valorativa; en definitiva, con un estado más elevado del ser en el que podemos dominar los estados aflictivos y orientarnos hacia un comportamiento más sabio, compasivo y ecuánime (Miró, 2006). 
Desde el punto de vista científico, el estado de mindfulness o de consciencia plena es el estado mental que emerge cuando se enfoca intencionalmente la atención hacia un soporte concreto (p.e. respiración, partes del cuerpo, objetos...), o abriendo la atención hacia los estímulos que proceden del exterior o de nuestro interior (Kaliman, 2017), mientras se observan sin apego los pensamientos, emociones y sensaciones físicas que surgen en el momento (Vago y Silberswig, 2012). Por ello el estado $M F$ implica, en primer lugar, una apertura mental a la experiencia (Bishop et al., 2004; Siegel, 2010) sin juicios ni valoraciones. Es decir, observar sin juzgar lo que está sucediendo, sin interponer el filtro de las propias creencias (Kabat-Zinn, 2003; Miró, 2006). Para ello, es necesario la aceptación de la experiencia tal cual se produce (Bishop et al., 2004; Kabat-Zinn, 2003; Miró, 2006). El estado de consciencia plena, pues, es ecuánime, ni nos aferramos ni rechazamos lo que sucede, ni comparamos, ni proyectamos. Dejamos pasar pensamientos y emociones, en una acción de soltar (Kabat-Zinn, 2003), de liberarnos de los mismos llevando el foco de atención a nuestras sensaciones físicas (u otros objetos de meditación). Nos mantenemos en el instante con humildad y curiosidad (Bishop et al., 2004; Siegel, 2010), con la mente de un principiante (Kabat-Zinn, 2003, 2009), pues cada momento es diferente al anterior. Asimismo, el estado de $M F$ nos conecta con el presente, se da en el aquí y el ahora (Bishop et al., 2004), dejando pasar pensamientos sobre el pasado (rumiaciones) o sobre el futuro (expectativas, temores, deseos).

Desde el punto de vista neurocientífico, mindfulness conlleva la disolución de las influencias del aprendizaje previo sobre la experiencia presente (Siegel, 2010). De acuerdo con Engel, Fries y Singer (2001), habitualmente procesamos la información de arriba abajo, pues las memorias de nuestras experiencias crean en nosotros un filtro que condiciona nuestra realidad e influencia nuestra percepción y comprensión del momento. Se trata de un proceso de clasificación de la nueva información sobre la base de categorías previas. Sin embargo, la información del presente, del aquí y el ahora, es mucho más amplia y rica que nuestra capacidad de procesarla de arriba abajo. La práctica de la atención plena nos da acceso a esa riqueza informativa del presente, reduciendo el procesamiento auto-referencial y aumentando la consciencia corporal (Tang, Hölzel y Posner, 2015). Llevar la atención a las corrientes sensoriales, junto con la observación de la experiencia, nos hace más conscientes de los procesos mentales y, por tanto, podemos orientar nuestras acciones superando la automaticidad del proceso ordinario.

El estado de consciencia plena implica un contacto directo con la realidad más allá del dualismo observador-observado, pues toda interpretación, análisis o construcción de significados produce la desambiguación de la realidad (Weimer, 1977). De acuerdo con Miró (2006),

las palabras traducen el mundo de la experiencia a una versión codificada que ya no es la experiencia. Cuando traducimos las experiencias en palabras, pasamos al plano conceptual [...] Pero en el plano de la experiencia [...] No hay separación. No hay evaluación, ni distinción. No hay observador, ni observado [...] Cuando sentimos algo como una sensación inmediata, directa, pura, estamos en atención plena o "mindfulness" (p. 36)

Así pues, mindfulness es un estado de consciencia no conceptual y no verbal, que carece de metas u objetivos, conectado a la experiencia directa del presente, sin filtros, juicios ni 
interpretaciones de nuestro ego, con carácter contemplativo y exploratorio más allá del dualismo observador-observado. Un estado que, a través de la práctica, puede mantenerse en el tiempo incorporándose a nuestro ser como un rasgo; es decir, la disciplina y la perseverancia en la práctica nos permiten conseguir y mantener un estado de "fluir atencional" de forma natural en el que se requiere menos esfuerzo (Ricard y Singer, 2018).

Finalmente, me gustaría insistir en que, desde su fundamento budista, mindfulness promueve la resiliencia y mejora la competencia social y emocional: la atención plena combinada con la empatía, la bondad y la compasión apoya la acción constructiva y el comportamiento afectuoso (Schoeberlein y Sheth, 2009). De este modo, la práctica o técnica meditativa que no incluye sus fundamentos morales y sus consecuencias hacia la acción política constructiva en el mundo, no es verdaderamente mindfulness.

\section{LA INVESTIGACIÓN POSITIVISTA EN PSICOLOGÍA SOBRE MINDFULNESS: ASPECTOS CRÍTICOS}

Las sociedades occidentales mantienen el materialismo como sistema de creencias hegemónico, según el cual, la realidad primaria del universo es la materia y, por tanto, todo tiene origen físico y se puede explicar en términos físicos (Taylor, 2018); es decir, no existe nada inmaterial ni metafísico. Consecuentemente, todo lo susceptible de ser investigado se reduce a aquello que se puede observar y verificar, manteniendo la distancia entre el observador y lo observado. Así pues, la cosmovisión científica materialista se basa en el positivismo, el dualismo, el determinismo -que permite predecir y controlar los sucesos- y el objetivismo -una supuesta objetividad al separar el observador del fenómeno observado- (Zohar y Marshall, 2001, siendo la principal fuente de legitimación del conocimiento y la verdad (Lyotard, 2000). Por ello las publicaciones científicas en general -y sobre mindfulness en particular- siguen siendo en su mayoría de carácter cuantitativo (Hyland, 2017).

Desde el positivismo, el objetivo de la investigación cuantitativa es la confirmación (o refutación) de una hipótesis (o un planteamiento teórico) mediante la obtención de unos datos numéricos supuestamente objetivos, a través de mediciones estandarizadas y controladas, con la intención de explicar un fenómeno y generalizar sus resultados, de modo que puedan extrapolarse a una población más amplia. Desde un punto de vista metodológico, la investigación científica positivista "divide" los fenómenos en variables medibles que, supuestamente, dan explicación de dicho fenómeno; algo así como el desmembramiento de un fenómeno en sus partes con la intención de que la suma de esas partes explique el todo. La ciencia positivista, pues, es el adalid de la visión del mundo objetivizada, cosificada, estructurada, medida, planificada, controlada y orientada al futuro, esto es, al resultado (Spira, 2019; Taylor, 2018). Con ello, se entiende, se construye un conocimiento válido, un régimen de verdad.

Así pues, la investigación positivista se basa en un marco definido a priori en el que tratamos de introducir los objetos de estudio, una plantilla conceptual y técnica para explicar la vida. Desde esta perspectiva, parece evidente que, si queremos estudiar mindfulness siendo fieles a su naturaleza esencial, la ciencia positivista nos puede dar poca luz. Si la atención plena es un estado de consciencia no dual, no conceptual, centrado en la experiencia misma, sin expectativas ni objetivos ¿cómo podemos definirla, medirla, manipularla, valorarla y (c) Psy, Soc, \& Educ, 2020, Vol. 12(1) 
compararla? ¿Cómo es posible acercarnos, si quiera un poco, a su esencia que es la naturaleza profunda de la mente, si la queremos estudiar desde los patrones de nuestra mente ordinaria dualista que clasifica, ordena, compara, mide y enjuicia?

Esta crítica que vierto en estos momentos se dirige al corazón de uno de los presupuestos fundamentales de la ciencia positivista: la validez. Sabido es que todo proceso científico de carácter positivista, es una reducción de la realidad hacia variables operativas que nos permitan cálculos y análisis estadístico. En este proceso de reducción, si queremos garantizar la rigurosidad del estudio, debemos ser fieles a la naturaleza del fenómeno a estudiar para asegurarnos que investigamos realmente lo que queremos investigar. Desde el comienzo del proceso investigativo positivista sobre $M F$, nos alejamos sucesivamente de su fundamento esencial $\mathrm{y}$, por tanto, no estamos investigando la atención plena, sino una serie de variables que, supuestamente, la definen pero que, en el mejor de los casos, sólo es un atisbo de su profundidad. El constructo mindfulness, por tanto, es inconsistente por la dificultad para acotarlo y medirlo, como también se ha observado en el constructo flow (v. Sparkes y Partington, 2003), y seguirá siendo así para la investigación psicológica positivista porque es incompatible con sus presupuestos.

El proceso de investigación cuantitativa en $M F$ lo desnaturaliza, por la propia perspectiva ontológica del paradigma positivista. Esta investigación se centra en el estudio de variables que pueden haber sido influenciadas con la práctica, es decir, lo instrumentaliza. Cuando intentamos repetir, mejorar, manipular o generalizar la práctica de la atención plena empezamos a desnaturalizarla. ¿Cómo es posible repetir y generalizar algo que de por sí es único e irrepetible? No dudo aquí de las bondades de estas investigaciones en la aplicación de la meditación en distintos problemas psicológicos, sino del hecho de que sea realmente mindfulness lo que se está investigando.

Asimismo, muchas investigaciones sobre la atención plena se han centrado en diferentes formas de meditación, con características y diseños de las prácticas muy variadas, lo cual dificulta la integración de los resultados respecto a un mismo fenómeno (Lutz, Jha, Dunne y Saron, 2015). En este sentido, la validez de constructo de los cuestionarios sobre atención plena que se usan profusamente en las investigaciones positivistas es más que problemática, dada esa variedad de prácticas englobadas en este término (Davidson y Kaszniak, 2015). Grossman (2011) es especialmente crítico con las escalas de medición de la atención plena pues sólo miden aspectos específicos de la misma, descontextualizan $M F$ de sus fundamentos éticos y actitudinales y, en términos generales, presentan una perspectiva adulterada de lo que es la mente. En este sentido, la práctica superficial de $M F$ así como gran parte de los procesos de investigación positivista, descuidan su fundamentos éticos, esto es, las conexiones entre la atención plena que nos permite observar el funcionamiento de nuestra mente y los fundamentos de la moralidad (Hyland, 2017). Además, como plantean Grossman y Van Dam (2011), en la definición y operacionalización de $M F$ no suele tenerse en cuenta la naturaleza y la progresión gradual de la estabilidad de la atención a través de la práctica, y tampoco la intensidad de la experiencia.

Otro de los aspectos problemáticos de la investigación psicológica sobre la atención plena remite a los diferentes resultados de la experiencia según sea la perspectiva de análisis, esto es, si es en primera, en segunda o en tercera persona. De acuerdo con Davidson y Kaszniak 
(2015), para una mejor comprensión de $M F$, así como para garantizar la validez y fiabilidad en las investigaciones, es necesario integrar al menos métodos de análisis de primera y tercera persona; es decir, obtener medidas de la experiencia en primera persona (autoinforme) y medidas en tercera persona de los procesos afectados por las variaciones en la experiencia, realizadas por un investigador sin relación previa con el sujeto.

Por otro lado, dada la variedad de posibles tradiciones, prácticas y procedimientos sobre $M F$ incluidos en los estudios científicos (Chiesa y Malinowski, 2011), un aspecto especialmente crítico es la interpretación y la integración de sus resultados. Para ello, sería necesaria una descripción profunda y detallada de las prácticas que siguen los participantes, así como mostrar en qué tradición o técnica específica se basan, con el objeto de comprender mejor el origen de la intervención y favorecer la integración de los resultados particulares del estudio en el contexto histórico y vital más amplio (Davidson y Kaszniak, 2015).

En definitiva, existe un gran número de inconsistencias en la investigación positivista sobre $M F$ respecto a su validez y fiabilidad, relativas a las propias prácticas y las formas de intervención, a los instrumentos de medición y al diseño de las investigaciones experimentales (v. Davidson y Kaszniak, 2015), que dificultan la comprensión de $M F$ por parte de la psicología clínica ya que la filosofía subyacente es no dualista (Singh Sikh y Spence, 2016). Junto a ello, la consideración de la consciencia plena como una mera técnica terapéutica, empobrece y limita su alcance al desatender sus implicaciones éticas y políticas, alejándola de su naturaleza original que no es otra que eliminar el sufrimiento en el mundo (Hyland, 2017).

\section{ALTERNATIVAS PARA LA INVESTIGACIÓN SOBRE MINDFULNESS: LA APERTURA AL PARADIGMA NATURALISTA}

Partiendo de la naturaleza no dual de la consciencia plena, es evidente que toda investigación sobre $M F$ implica, inevitablemente, una reducción de la realidad y un acercamiento parcial al fenómeno. No obstante, como apunté en el apartado anterior, la investigación psicológica de carácter positivista presenta demasiadas limitaciones dado el posicionamiento ontológico del que parte. En este sentido, mindfulness remite a una ontología en la que la esencia de la realidad no es material, sino espiritual. Por ello, es fundamental asumir una perspectiva científica ontológica posmaterialista en las investigaciones sobre la consciencia plena. Al respecto, es necesario aclarar que, de acuerdo con Taylor (2018), existe una confusión en el mundo científico hegemónico cuando se rechazan otras vías de conocimiento, otras formas de racionalidad y, por tanto, otras formas de investigar, pues ser racional no equivale a adscribirse a la concepción materialista del mundo. De este modo,

el posmaterialismo sostiene que la materia no es la realidad primaria del universo, y que fenómenos como la consciencia y la vida no pueden explicarse por completo recurriendo a la biología y la neurología. El posmaterialismo defiende que hay algo más fundamental que la materia, algo que puede designarse de distintas maneras: mente, consciencia o espíritu (Taylor, 2018, p. 11).

Esta posición ontológica no materialista, nos brinda el acceso a la subjetividad, al dinamismo y al cambio propios de la experiencia humana, lo que nos permite dialogar e integrar los presupuestos de la cosmovisión oriental, con el fin de dotar de rigor científico las investigaciones sobre $M F$ (Singh Sikh y Spence, 2016); esto es, buscar la coherencia entre su (c) Psy, Soc, \& Educ, 2020, Vol. 12(1) 
naturaleza ontológica y los métodos de investigación. En esta línea, el paradigma naturalista o interpretativo parte de una naturaleza de la realidad subjetiva y múltiple a la que las personas damos significado desde nuestra propia experiencia. Asimismo, las investigaciones naturalistas no sólo están enfocadas hacia los resultados, sino que el estudio del propio proceso es un pilar clave para conocer más profundamente el fenómeno estudiado. Epistemológicamente, no existe la dualidad observador/observado, en tanto la dimensión subjetiva está presente en el centro de la investigación; no hay, pues, una separación entre el investigador y el objeto de investigación en la búsqueda no de la explicación sino de la comprensión del estado de mindfulness. Desde este punto de vista, parece claro que el investigador, aquí, necesita acercarse a la consciencia plena desde la propia experiencia, lo que le permitirá discernir e interpretar con mayor rigor los hallazgos de sus investigaciones con otras personas.

Puesto que la atención plena es un estado del ser humano en su globalidad, sin duda puede ser explorada desde múltiples enfoques de investigación. Por ello, más allá de la visión particular que un área científica puede aportar, necesitamos de "un diálogo entre disciplinas que antes se encontraban aisladas, como la neurociencia, la psicología del desarrollo, la psicoterapia y el propio conocimiento de la mente (mindsight) que se alcanza con la práctica de mindfulness" (Simón, 2006, p. 7), así como las ciencias sociales. Del mismo modo, es recomendable la apertura a investigaciones naturalistas y métodos hermenéuticos basados en filosofías no dualistas (Singh Sikh y Spence, 2016), que nos permitan integrar sus potenciales. Mindfulness no refiere a la mente ordinaria, es decir, no estamos hablando de un fenómeno de pensamiento, sino de un estado de calma mental y atención plena, en el cual observamos lo que sucede en nuestro interior permitiendo que emerjan ideas desde nuestra sabiduría interna, desde la consciencia no condicionada. Por ello, la investigación naturalista se acerca mucho más a su esencia y a lo que podemos aprender de este estado, esto es, entender mejor la realidad en la que vivimos y a nosotros mismos.

Singh Sikh y Spence (2016) realizan un interesante proceso de conexión entre las filosofías hermenéuticas occidentales y las orientales que aporta un mayor acercamiento a la naturaleza ontológica de $M F$, y nos muestra un método de investigación más coherente. Desde el punto de vista oriental, los seres humanos son parte de la experiencia, una visión similar a la noción de "Ser" de Heidegger que disuelve la distancia entre sujeto y objeto, y abre las puertas de la hermenéutica ontológica. Desde esta cosmovisión no dualista, siguiendo a Singh Sikh y Spence (2016), la comprensión y el conocimiento se obtienen a partir de la verdad que emerge de la unidad ontológica de la experiencia, en la cual la persona y el fenómeno investigado son partes de una misma realidad. Por tanto, para alcanzar la experiencia existencial, la persona debe suspender todas las dualidades, incluidas las creadas por el propio pensamiento y el lenguaje. Por otro lado, puesto que la mente ordinaria nos aporta una visión dual y condicionada que oscurece la realidad, la comprensión no condicionada profunda surge de la consciencia pura o primordial, a la que sólo podemos acceder a través de la atención plena en el momento presente. Así pues, la meditación misma se convierte en una parte crítica del proceso de comprensión de $M F$, pues las interpretaciones auténticas surgen de los insights que provienen de la consciencia no condicionada.

Este planteamiento tiene unas interesantes aplicaciones en el ámbito de la investigación psicológica sobre $M F$. La primera de ellas, como apunté anteriormente, es que un factor clave 
para la comprensión de la atención plena reside en la propia práctica, en cómo se desarrolla el proceso. Por ello, es esencial que los investigadores interesados sean también practicantes habituales de esta meditación y tengan experiencia suficiente. Como escribe Sogyal-Rimpoché (1996), para comprender la consciencia plena "hace falta la madurez que solo se obtiene con años de escuchar, contemplar, reflexionar, meditar y practicar de manera constante" (p. 100).

Asimismo, creo necesario acometer más investigaciones de carácter cualitativo a través de entrevistas abiertas que nos reporten una información más profunda sobre la experiencia del participante, así como su repercusión en el conjunto de su vida, atendiendo no sólo las posibles mejoras respecto a problemas psicológicos concretos, sino en lo relativo a sus valores y actitudes morales. De acuerdo, con Grossman y Van Dam (2011), en las intervenciones basadas en la atención plena es necesario considerar las consecuencias y los desafíos morales que implican el uso de las técnicas de meditación respecto al aumento de la consciencia sobre la realidad que nos rodea.

El énfasis en el análisis global, la atención a la experiencia desde una perspectiva holística y multidisciplinar, así como los constantes procesos de corroboración, triangulación y adecuación referencial, señalan el gran potencial que el paradigma naturalista puede aportar a la investigación sobre mindfulness. De acuerdo con Crossley (2000), la psicología narrativa reconoce que el lenguaje hace que la experiencia sea significativa, por lo que ocupa un papel central en la construcción de la identidad. De este modo, podemos acercarnos de una manera más rigurosa y comprensiva a la naturaleza de este fenómeno mediante la conexión de las narrativas de los participantes sobre los insights y efectos de su práctica, con su contexto vital y su evolución personal (Singh Sikh y Spence, 2016), yendo más allá de la medición únicamente de sus efectos sobre diferentes trastornos.

\section{CONCLUSIONES}

La amplia aceptación de mindfulness en las sociedades occidentales y, especialmente, en el ámbito científico y académico, supone una interesante y enriquecedora oportunidad para acercar los presupuestos ontológicos y epistemológicos de las filosofías orientales (particularmente, del budismo) y occidentales. Sin embargo, como he mostrado a lo largo de este artículo, existe una prevalencia de las investigaciones positivistas sobre la atención plena que se distancian de su naturaleza ontológica, y desatienden sus fundamentos morales e implicaciones políticas. Con ello, el alcance de la comprensión de la consciencia plena, así como el desarrollo de sus grandes potenciales para el bienestar personal y la integración de actitudes morales que mejoran la convivencia, son limitados y empobrecidos. El paradigma científico naturalista y los métodos cualitativos de investigación, así como la propia práctica de la meditación, son vías que podrían acercarnos mejor a su profundidad espiritual, y ayudarnos a abrir nuestra perspectiva sobre la naturaleza de la realidad y del ser humano.

No obstante, he de insistir en que no es mi intención cuestionar aquí las bondades de la práctica de la meditación respecto a los distintos trastornos psicológicos, ni los efectos probados científicamente sobre los mismos, pues una herramienta que puede aportar beneficios a las personas no puede tener una connotación negativa. Sin embargo, es necesario aclarar que, en estos casos, no estamos investigando realmente sobre mindfulness en toda su amplitud y profundidad, siendo fieles a su naturaleza esencial. Estamos hablando, efectivamente, de una (c) Psy, Soc, \& Educ, 2020, Vol. 12(1) 
determinada técnica terapéutica. Por ello, considero fundamental definir dicha técnica de una manera específica propia, así como concretar en qué consiste exactamente su práctica como medio terapéutico, tratando de superar la ambigüedad que supone su acrítica inclusión en el amplio y genérico paraguas de la denominada atención plena.

\section{REFERENCIAS}

Alvear, D. (2015). Mindfulness en positivo. La ciencia de la atención plena y la psicología positiva en el camino del bienestar. Lleida: Milenio.

Bishop, S. R., Lau, M. A., Shapiro, S. L., Carlson, L. E., Anderson, N. D., Carmody, J., Segal, Z., Abbey, S., Speca, M., Velting, D. y Devins, G. (2004). Mindfulness: A proposed operational definition. Clinical Psychology: Science and Practice, 11(3), 230-242. https://doi.org/10.1093/clipsy.bph077

Campos, D., Cebolla, A., Quero, S., Bretón-López, J., Botella, C., Soler, J., García-Campayo, J., Demarzo, M., y Baños, R. M. (2016). Meditation and happiness: mindfulness and self-compassion may mediate the meditation-happiness relationship. Personality and Individual Differences, 93, 80-85. http://dx.doi.org/10.1016/j.paid.2015.08.040

Cebolla, A. y Campos, D. (2016). Enseñar mindfulness: contextos de instrucción y pedagogía. Revista de Psicoterapia, 27(103), 103-118.

Cebolla, A., Enrique, A., Alvear, D., Soler, J., y García-Campayo, J. (2017). Psicología positiva contemplativa: integrando mindfulness en la psicología positiva. Papeles del Psicólogo, 38(1), 12-18. https://doi.org/23923 /pap.psicol2017.2816

Chiesa, A., Calati, R. y Serretti, A. (2011). Does mindfulness training improve cognitive abilities? A systematic review of neuropsychological findings. Clinical Psychology Review, 31(3), pp.449-464. https://doi.org/10.1016/j.cpr.2010.11.003

Chiesa, A. y Malinowski, P. (2011). Mindfulness-based approaches: Are they all the same? Journal of Clinical Psychology, 67, 404-424. https://doi.org/10.1002/jclp.20776

Crossley, M. (2000). Introducing narrative psychology. Buckingham, UK: Open University Press.

Davidson, R.J. y Kaszniak, A.W. (2015). Conceptual and Methodological Issues in Research on Mindfulness and Meditation. American Psychologist, 70(7), 581-592. https://doi.org/10.1037/a0039512

Engel, A.K., Fries, P. y Singer, W. (2001). Dynamic predictions: Oscillations and synchrony in top-down processing. Nature Reviews Neuroscience, 2, 704-716. https://doi.org/10.1038/35094565

Goleman, D. y Davison, R. J. (2017). Los beneficios de la meditación. Barcelona: Kairós.

Grossman, P. (2011). Defining mindfulness by how poorly I think I pay attention during everyday awareness and other intractable problems for psychology's (re)invention of mindfulness: comment on Brown et al. (2011). Psychological Assessment, 23, 10341040. http://dx.doi.org/10.1037/a0022713

Grossman, P. y Van Dam, T. (2011). Mindfulness, by any other name... Trials and Tribulations of Sati in Western Psychology and Science. Contemporary Buddhism, 12, 219-239. https://doi.org/10.1080/14639947.2011.564841 
Hervás, G, Cebolla, A. y Soler, J. (2016). Intervenciones psicológicas basadas en mindfulness y sus beneficios: estado actual de la cuestión. Clínica y Salud, 27, 115-124. http://dx.doi.org/10.1016/j.clysa.2016.09.002

Hyland, T. (2017). McDonaldizing Spirituality: Mindfulness, Education and Consumerism. Journal of Transformative Education, 15(4), 334-356. https://doi.org/10.1177/1541344617696972

Kabat-Zinn, J. (2003). Mindfulness-based interventions in context: Past, present, and future. Clinical Psychology: Science and Practice, 10(2), 144-156. https://doi.org/10.1093/clipsy.bpg016

Kabat-Zinn, J. (2009). Mindfulness en la vida cotidiana. Donde quiera que vayas, ahí estás. Madrid: Paidós.

Kaliman, P. (2017). La ciencia de la meditación. Barcelona: Kairós.

Keng, S. L., Smoski, M. J., y Robins, C. J. (2011). Effects of mindfulness on psychological health: A review of empirical studies. Clinical Psychology Review, 31, 1041-1056. https://doi.org/10.1016/j.cpr.2011.04.006

Linares, L., Estévez, A., Soler, J. y Cebolla, A. (2016). El papel del mindfulness y el descentramiento en la sintomatología depresiva y ansiosa. Clínica y Salud, 27, 51-56. http://dx.doi.org/10.1016/j.clysa.2016.03.001

Lutz, A., Jha, A., Dunne, J.D. y Saron, C.D. (2015). Investigating the phenomenological matrix of mindfulness-related practices from a neurocognitive perspective. American Psychologist, 70, 632-658. https://doi.org/10.1037/a0039585

Lyotard, F. (2000). La condición posmoderna. Madrid: Cátedra.

Mañas, I. (2009). Mindfulness (Atención Plena): La meditación en psicología clínica. Gaceta de Psicología, 50, 13-29.

Miró, M.T. (2006). La atención plena (mindfulness) como intervención clínica para aliviar el sufrimiento y mejorar la convivencia. Revista de psicoterapia, 17 (66-67), 31-76.

Ricard, M. y Singer, W. (2018). Cerebro y meditación. Diálogo entre el budismo y las neurociencias. Barcelona: Kairós.

Ruiz, L.J. y Callejón, Z. (2014). Terapias conductuales de tercera generación en personas mayores: una revisión de la literatura. Psychology, Society, \& Education, 6(2), 113-124.

Sansó, N., Galiana, L., Oliver, A., Cuesta, P., Sánchez, C. y Benito, E. (2018). Evaluación de una Intervención Mindfulness en Equipos de Cuidados Paliativos. Psychosocial Intervention, 27(2), 81-88. https://doi.org/10.5093/pi2018a7

Schoeberlein, D. y Sheth, S. (2009). Mindful teaching and teaching mindfulness. Somerville, MA: Wisdom.

Siegel, D. J. (2010). Mindsight. Oxford: Oneworld.

Simón, V. (2006). Mindfulness y neurobiología. Revista de psicoterapia, 17(66-67), 5-30.

Simón, V. (2014). El reencuentro científico en la compasión. En A. Cebolla, J. GarcíaCampayo y M. Demarzo (Eds.), Mindfulness y ciencia (pp. 191-225). Madrid: Alianza.

Singh Sikh, B. y Spence, D. (2016). Methodology, Meditation, and Mindfulness: Toward a Mindfulness Hermeneutic. International Journal of Qualitative Methods, 15(1). 1-8. https://doi.org/10.1177/1609406916641251 
Sogyal-Rimpoché (1996). Destellos de sabiduría. Reflexiones sobre la vida y la muerte. Barcelona: Urano.

Sogyal-Rimpoché (2006). El libro tibetano de la vida y la muerte (ed. revisada y actualizada). Barcelona: Urano.

Sparkes, A.C. y Partington, S. (2003). Narrative practice and its potential contribution to sport psychology: The example of flow. The Sport Psychologist, 17(3), 292-317. http://dx.doi.org/10.1123/tsp.17.3.292

Spijkerman, M. P. J., Pots, W. T. M. y Bohlmeijer, E. T. (2016). Effectiveness of online mindfulness-based interventions in improving mental health: A review and metaanalysis of randomized controlled trials. Clinical Psychology Review, 45, 102-114. http://dx.doi.org/10.1016/j.cpr.2016.03.009.

Spira, R. (2019). La naturaleza de la conciencia. Barcelona: La Llave.

Strauss, C., Cavanagh, K., Oliver, A. y Pettman, D. (2014). Mindfulness-based interventions for people diagnosed with a current episode of an anxiety or depressive: A meta-analysis of randomized controlled trials. PLoS One, 9(4), e96110. http://dx.doi.org/10.1371/journal.pone.0096110

Taylor, S. (2018). Por una ciencia espiritual. Badalona: Koan.

Tang, Y. Y., Hölzel, B. K. y Posner, M. I. (2015). The neuroscience of mindfulness meditation. Nature Reviews Neuroscience, 16, 213-225. http://dx.doi.org/10.1038/nrn3916

Vago, D. R. y Silbersweig, D. A. (2012). Self-awareness, self-regulation, and selftranscendence (S-ART): a framework for understanding the neurobiological mechanisms of mindfulness. Frontiers in Human Neuroscience, 6, 296. https://doi.org/10.3389/fnhum.2012.00296

Weimer, W. B. (1977). A conceptual framework for cognitive psychology: the motor theories of the mind. En R. Shaw y J. Bransford (eds), Perceiving, Acting and Knowing (pp. 267311). Hillsdale: LEA.

Williams, J.M.G., y Kabat-Zinn, J. (2013). Mindfulness: Diverse perspectives on its meaning, origins and applications. Abingdon: Routledge.

Yagüe, L., Sánchez-Rodríguez, A.I., Mañas, I., Gómez-Becerra, I. y Franco, C. (2016). Reducción de los síntomas de ansiedad y sensibilidad a la ansiedad mediante la aplicación de un programa de meditación mindfulness. Psychology, Society, \& Education, 8(1), 23-37.

Zohar, D. y Marshall, I. (2001). Inteligencia espiritual. Barcelona: Plaza y Janés. 